\title{
Agreement of Immunoassay and Tandem Mass Spectrometry in the Analysis of Cortisol and Free T4: Interpretation and Implications for Clinicians
}

\author{
Rochelle E. Tractenberg, ${ }^{1}$ Jacqueline Jonklaas, ${ }^{2}$ and Steven J. Soldin ${ }^{2,3}$ \\ ${ }^{1}$ Departments of Neurology; Biostatistics, Bioinformatics \& Biomathematics and Psychiatry, \\ Georgetown University Medical Center, Washington, DC 20007, USA \\ ${ }^{2}$ Division of Endocrinology, Georgetown University Medical Center, Washington, DC 20007, USA \\ ${ }^{3}$ Bioanalytic Core Laboratory, General Clinical Research Center, and Departments of Pharmacology and Medicine, \\ Georgetown University Medical Center, Washington, DC 20007, USA
}

Correspondence should be addressed to Rochelle E. Tractenberg, ret7@georgetown.edu

Received 3 December 2009; Accepted 29 April 2010

Academic Editor: Steen Honoré Hansen

Copyright ( $\odot 2010$ Rochelle E. Tractenberg et al. This is an open access article distributed under the Creative Commons Attribution License, which permits unrestricted use, distribution, and reproduction in any medium, provided the original work is properly cited.

Objective. To quantify differences in results obtained by immunoassays (IAs) and tandem mass spectrometry (MSMS) for cortisol and free thyroxine (FT4). Design \& Patients. Cortisol was measured over 60 minutes following a standard ACTH stimulation test $(n=80)$; FT4 was measured over time in two cohorts of pregnant $(n=57)$, and nonpregnant $(n=28)$ women. Measurements. Samples were analyzed with both IA and MSMS. Results. Results for cortisol by the two methods tended to agree, but agreement weakened over the 60-minute test and was worse for higher (more extreme) concentrations. The results for FT4 depended on the method. IA measurements tended to agree with MSMS measurements when values fell within "normal levels", but agreement was not constant across trimester in pregnant women and was poorest for the extreme (low/high) concentrations. Correlations between MSMS measurements and the difference between MSMS and IA results were strong and positive $(0.411<r<0.823$; all $P<.05)$. Conclusions. IA and MSMS provide different measures of cortisol and FT4 at extreme levels, where clinical decision making requires the greatest precision. Agreement between the methods is inconsistent over time, is nonlinear, and varies with the analyte and concentrations. IA-based measurements may lead to erroneous clinical decisions.

\section{Introduction}

In this paper, we compare the agreement [1-7] of immunoassay (IA) and tandem mass spectrometry (MSMS) in the measurement of the concentrations of two analytes, cortisol, and free T4. Both analytes are critical for normal growth and development, and survival. Thyroid hormone regulates the expression of multiple genes and is necessary to maintain normal function in virtually all organ systems of the body [8].

Cortisol regulates normal responses to stress, and is important for vascular reactivity, carbohydrate metabolism, and immune function [9]. Cortisol measurement is the basis for diagnosing adrenal insufficiency or conditions of glucocorticoid excess. Mismeasurement of cortisol could lead to withholding treatment for patients with adrenal insufficiency or Cushings syndrome, which could have life-threatening consequences. Similarly, incorrect diagnosis of these conditions exposes patients to the toxic side effects of steroid or steroid-lowering therapies, without any expected benefit. FT4 is one of the analytes used to titrate the treatment of hypothyroidism and hyperthyroidism. Erroneous analyte values could lead to mismanagment of these disorders. This is a particular concern in vulnerable populations such as the young, the elderly, those who are pregnant, those suffering from cardiac disease, or those who have thyroid cancer. Although FT4 is interpreted in conjunction with a thyroid stimulating hormone (TSH) level, there are circumstances under which TSH measurements are unreliable [10]. It has also been suggested that during pregnancy a decreased FT4 
Table 1: Descriptive statistics for cortisol (Table 1(a)) and Free T4(Table 1(b)).

(a) Cortisol $(\mathrm{mcg} / \mathrm{dl})$ over a 60 minute test period by MS-MS and IA $(N=$ 80). Note that agreement is not universal on reference ranges. However, Reference range for random cortisol by IA in most clinical laboratories $=4$ $-22 \mathrm{mcg} / \mathrm{dl}$ ([23]; see also [24]).

\begin{tabular}{lll}
\hline & MSMS: MEAN (SD) & IA: MEAN (SD) \\
\hline cortisol $T=0$ & $9.498(6.08)$ & $8.508(4.99)$ \\
cortisol $T=30$ & $21.982(10.24)$ & $20.553(8.96)$ \\
cortisol $T=60$ & $25.758(12.22)$ & $23.844(10.74)$ \\
\hline
\end{tabular}

(b) Free $\mathrm{T} 4$ (FT4, ng/dl) over 3 trimesters of pregnancy, and in nonpregnant women. Note that agreement on reference ranges for FT4 in pregnant women is not universal. Kahric-Janicic et al. (2007) [25] suggest ranges between 0.6 and $1.4 \mathrm{ng} / \mathrm{dL}$ for FT4 in pregnant women; results vary by trimester for both MSMS and IA. Reference intervals for FT4 in nonpregnant women by tandem mass spectrometry are $0.8-2.1 \mathrm{ng} / \mathrm{dL}$ [24]. Ranges for this analyte in nonpregnant women by IA are lower and have a smaller range, typically around $0.7-1.5 \mathrm{ng} / \mathrm{dL}$ [26].

\begin{tabular}{lll}
\hline & $\begin{array}{l}\text { MSMS: MEAN } \\
(\text { SD), N }\end{array}$ & IA: MEAN (SD), N \\
\hline FT4, trimester 1 & $1.125(.23), 59$ & $1.071(.22), 61$ \\
FT4, trimester 2 & $0.915(.31), 36$ & $0.795(.17), 42$ \\
FT4, trimester 3 & $0.863(.22), 26$ & $0.875(.18), 35$ \\
$\begin{array}{l}\text { FT4, nonpregnant } \\
\text { women }\end{array}$ & $0.928(.26), 28$ & $1.102(.25), 28$ \\
\hline
\end{tabular}

concentration, not an elevated TSH, specifically places a fetus at risk [11-13]. Thus accurate determination of FT4 will best serve the clinician who wishes to make an informed clinical decision.

Direct/analogue immunoassay (IA) methods for the measurement of FT4 are widely used and are controversial [14-17], and there is discrepancy between different IAs [18-20]. Recent reports of problems with IAs for the measurement of steroids in general have identified lack of specificity as the key cause of unreliability [21, 22]. In this paper, we compare measurements of cortisol and FT4 concentrations made by IA and MSMS plotting the mean versus the difference between two measures of a single analyte. It is important to note here that we seek to quantify the agreement between the two measurement methods and/or their disagreement, and not quantify or correct for the degree of disagreement.

\section{Methods}

2.1. Overview. This study represents secondary analyses of previously existing data $[24,25]$. There were two different cohorts for whom analyte measurements were already available. Briefly, the cohort for whom cortisol measurements were available consisted of 80 subjects [24] who underwent an outpatient cortrosyn stimulation test as part of an evaluation for adrenal insufficiency [27, 28]. The reference interval for cortisol is $3-21 \mathrm{mg} / \mathrm{dL}$ (Soldin SJ, unpublished data). FT4 measurements were available for 28 clinically and biochemically euthyroid nonpregnant women and 98 clinically and biochemically euthyroid pregnant women who underwent testing during one or more trimesters of pregnancy [25]. The reference interval for FT4 is 0.9$1.6 \mathrm{ng} / \mathrm{dL}$ in nonpregnant women which is the same as the pediatric reference range [29]. We compared assay results from IA and MSMS using the means-difference plots. We would conclude that the methods agree if we observe no relationship between data points plotted on these axes [2-4].

2.2. Samples. Samples for cortisol and FT4 were collected in plastic red top tubes (containing clot activator, Vacutainer, manufactured by Becton Dickenson, Franklin Lakes, NJ 07417) and allowed to clot for 20 minutes. The samples were then centrifuged at $4,000 \mathrm{rpm}$ for $10 \mathrm{~min}$, serum separated and immediately stored at minus $80^{\circ} \mathrm{C}$ until measurement of the analytes by the General Clinical Research Center Bioanalytical Core Laboratory. Cortisol concentrations are reported in $\mu \mathrm{g} / \mathrm{dL}$ at baseline and then 30 minutes and 60 minutes after cortrosyn injection. This diagnostic test, which involves injection of synthetic adrenocorticotropic stimulating hormone (cortrosyn), is a common test for diagnosing adrenal insufficiency in clinical situations [28]. FT4 measurements were obtained either in the nonpregnant state or during successive trimesters of pregnancy and are reported in $\mathrm{ng} / \mathrm{dL}$.

\subsection{Assays}

2.3.1. Immunoassays. Cortisol was measured on the DPC Immulite 1000 (Diagnostic Products Corporation, Los Angeles, CA) while FT4s were measured on the Dade RxL Dimension (Dade-Behring Diagnostics, Glasgow, DE).

2.3.2. LC/MS/MS. Both the cortisol and FT4s were assayed as previously published in $[17,20-22]$. Both assays were performed using the API-5000 tandem mass spectrometer (Applied Biosystems, Foster City, CA), and both use deuterated internal standards. Cortisol measurement was performed on $200 \mu \mathrm{L}$ serum [22], while FT4 was measured on a serum ultrafiltrate $[17,20]$. Assay values (concentrations of cortisol or FT4) were obtained via IA and MSMS at each of the time points relevant for the two analytes.

2.3.3. Statistical Methods. Data in each study cohort were analyzed separately using a Bland-Altman (BA) or meansdifference plot for the IA-MSMS pairs of measurements. The "summary" of data in the BA plot is reflected in reference lines at zero, representing the ideal mean difference between the two measures, and the values one standard deviation (solid lines) and two standard deviations (short dashed lines) away from zero (long dashed line) on the $Y$ axis, with zero being the point on the $Y$ axis where the two methods agree perfectly and the lines within one standard deviation bounding the acceptable range for variability in agreement. Pearson correlation coefficients were computed to determine if there was a significant association between 


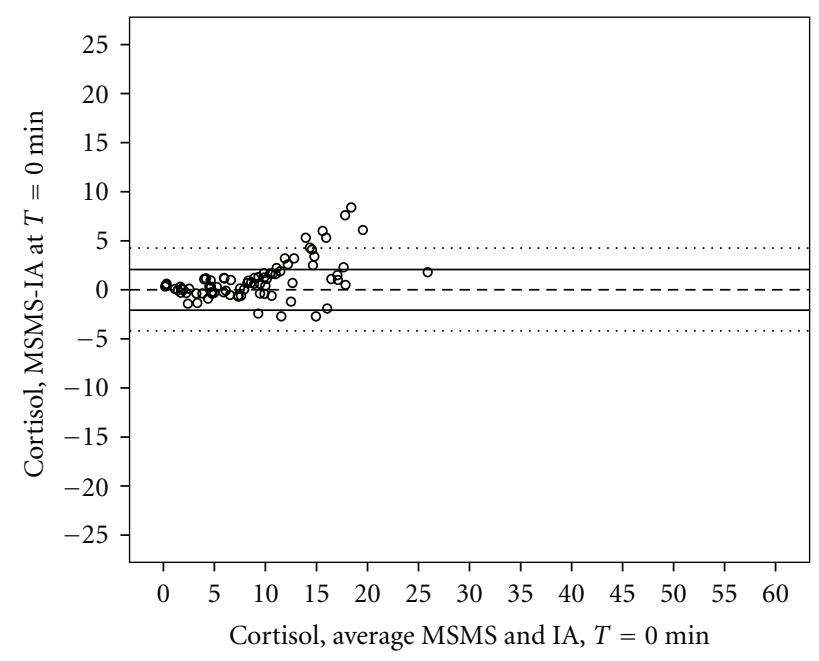

(a)

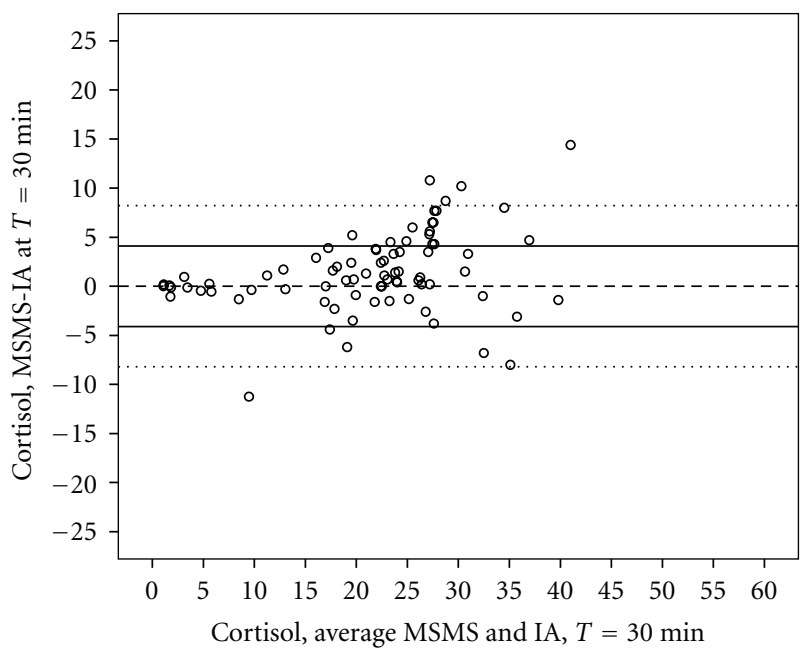

(b)

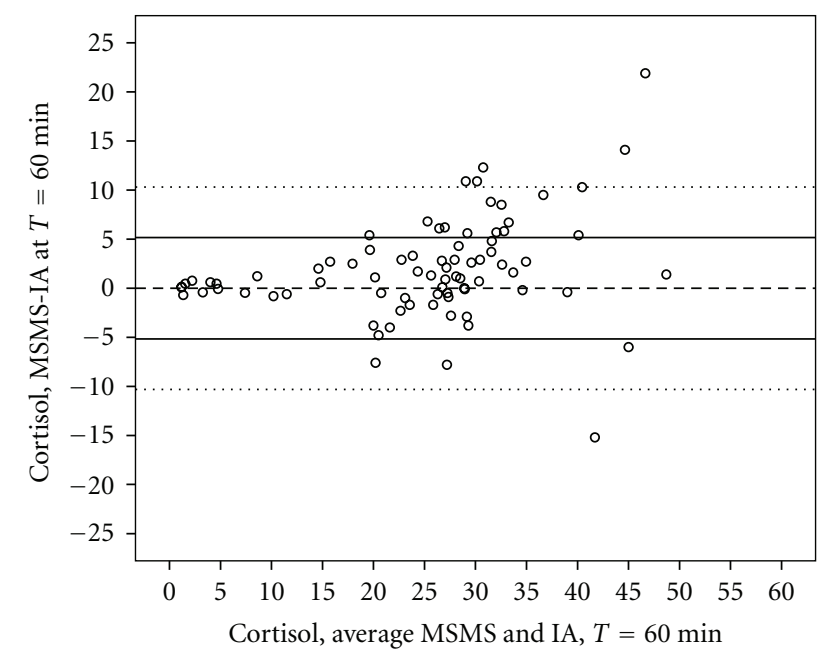

(c)

FIGURE 1: Bland-Altman plot of Cortisol (( $\mathrm{mcg} / \mathrm{dl})$ at $T=0(\mathrm{a}), T=30$ (b), and $T=60$ (c) minutes) in the cortisol response test: difference MSMS-IA on $Y$ axis and average on $X$ axis. $N=80$. Reference line at zero and pairs of lines at \pm 1 SD and \pm 2 SD.

the difference between measurements (MSMS-IA) and one of the measurements (MSMS), which would reflect significant disagreement [7].

\section{Results}

The data are shown first for cortisol values at baseline and then 30 and 60 minutes after cortrosyn injection and then for free $\mathrm{T} 4$ values sampled in the nonpregnant state and then during the successive trimesters of pregnancy. Tables $I(a)$ and I(b) present descriptive statistics for cortisol (1A) and FT4 (1B) across the relevant time points.

3.1. Cortisol. Figure 1 shows the Bland-Altman (BA) plot of the IA and MSMS measurements for the cortisol measurements initially (T0, Figure 1(a)), at 30- (T30, Figure 1(b)) and 60-minutes (T60, Figure $1(\mathrm{c})$ ).
A clear trend in increasing variance is observed along the $X$-axis (representing the mean of the two measures). Lower concentrations of cortisol measured by IA or MSMS were fairly closely associated, but as the values increase, the divergence becomes larger. This heteroscedasticity within the data at the 30 minute point of the cortisol response test is larger, and reflects a larger range of cortisol values, than were observed at baseline (Figure 1(a)). The same tendency towards increasing variance is apparent in Figure 1(c) $(60$ minutes after the injection).

Table 2 presents the correlation coefficients estimating the strength of association between the difference between the two measures of cortisol and the MSMS results for cortisol. MSMS results are strongly and positively correlated with the difference.

3.2. FT4. Figure 2 shows the Bland-Altman (BA) plots of the IA and MSMS measurements for FT4 measured in the first 


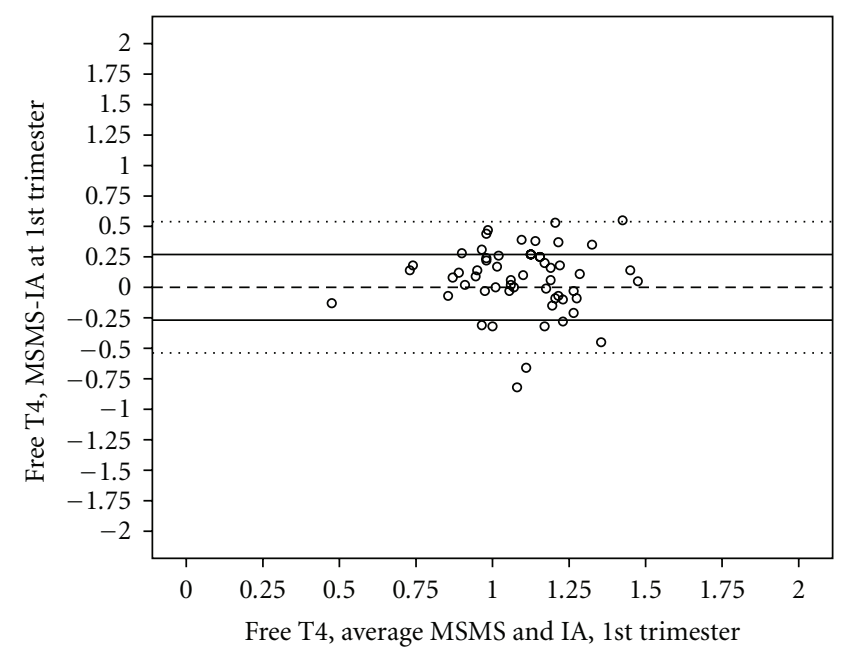

(a)

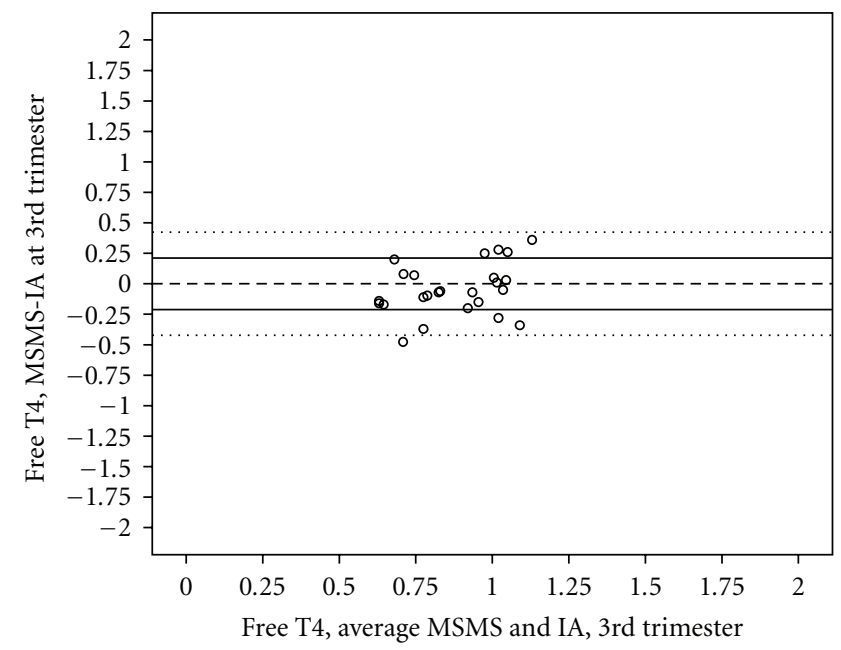

(c)

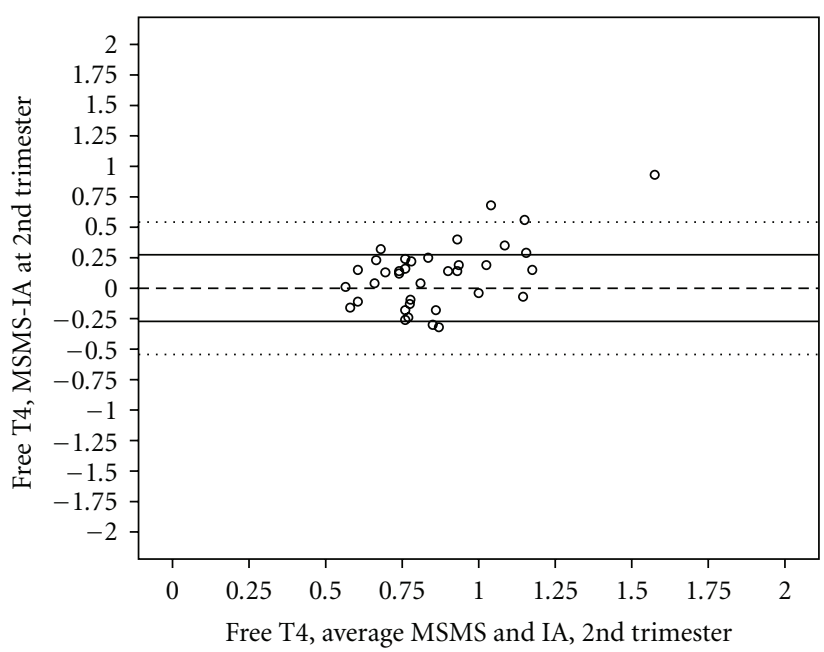

(b)

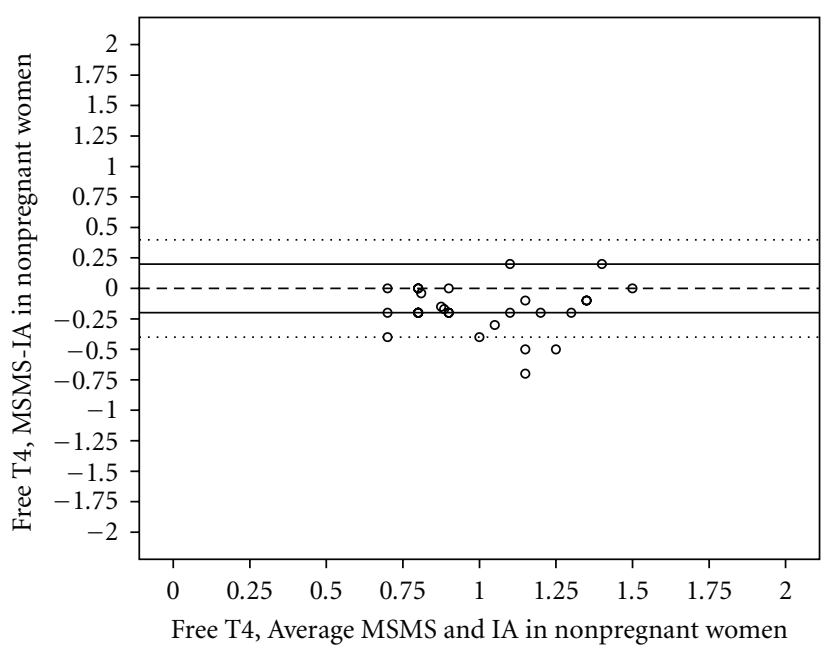

(d)

FIgURE 2: Bland-Altman plots of FT4 (ng/dl), first trimester (T1, 2A), second trimester (T2, 2B), 3rd trimester (T3, 2C), and in nonpregnant (NP, 2D) women. Difference (MSMS-IA) on Y axis and average value from IA and MSMS on X axis. (Pregnant cohort N with both ranges from 59 (T1) to 26 (T3); nonpregnant cohort $\mathrm{N}$ is 28.) Reference line at zero and pairs of lines at $\pm 1 S D$ and $\pm 2 S D$.

TABle 2: Pearson correlations of difference between methods versus MSMS alone, for Cortisol $(N=80)$. MSMS and (MSMS-IA) correlations reflect significant differences in the variances of the measurements by IA versus MSMS over time.

\begin{tabular}{llll}
\hline DIFFERENCE & $\begin{array}{l}\text { Cortisol by MSMS, } \\
T=0\end{array}$ & $\begin{array}{l}\text { Cortisol by MSMS, } \\
T=30 \text { min }\end{array}$ & $\begin{array}{l}\text { Cortisol by MSMS, } \\
T=60 \text { min }\end{array}$ \\
\hline$T=0$ min, MSMS-IA & $0.650^{*}$ & & $0.494^{*}$ \\
$T=30$ min, MSMS-IA & & & $0.480 *$ \\
$T=60$ min, MSMS-IA & & & \\
\hline
\end{tabular}

All Pearson correlation coefficients significant at $P \leq .001 .{ }^{*}$ indicates significant differences in the variances of the measurements by IA versus MSMS.

trimester (T1, 2A), second (T2, 2B) and third trimesters (T3, 2C) for a single group of pregnant women followed over time, and a fourth group of women who were not pregnant (NP, 2D).

The patterns in the means-difference plots are not as clear cut for FT4 as for cortisol. In the first trimester (T1, Figure $2(\mathrm{a}))$, the variability in disagreement appears to increase as the average increases (fan shape), while in T2 and T3 the difference itself seems to increase as the average increases (positive slope). For the nonpregnant women, a different pattern is observed, with the majority of points reflecting greater FT4 values from IA relative to those from MSMS.

Table 3 presents the correlation matrix for the difference in FT4 measured by MSMS and IA with MSMS. 
Strong, positive correlations were observed between the difference between the two methods and MSMS measurements of FT4 over the three trimesters and in the nonpregnant state, suggesting that the patterns reflected in Figures 2(a)-2(d) represent significant differences in the variances of the measurements of FT4 by IA versus MSMS.

\section{Discussion and Conclusions}

This study of two methods to assay FT4 and cortisol over time showed nonlinear disagreement between analytes measured by immunoassay and tandem mass spectrometry. The differences were more dramatic for cortisol than for FT4 but significant correlation coefficients reflected "genuine" trends of increasing variance associating with increased analyte concentration, and over time, for both analytes. In these correlations, we treated MSMS as the standard measurement; IA results are derived through a mathematical formula in the direct/analogue methods used in the great majority ( $>99 \%$ ) of clinical laboratories.

Our results suggest significant variation (heteroscedasticity) and nonequivalence of these two methods. This could simply reflect poor reliability in IA for these analytes but in the case of FT4 it is also perhaps suggestive that the variation in disagreement over trimesters could also have varying causes (i.e., that vary with pregnancy, e.g., heterophilic antibodies, changes in protein binding, etc.). Although the agreement was worse for cortisol, correlations between the difference in results from the two methods and MSMS was significant for both analytes and at each of the time points.

The purpose of this work is not to provide correct/corrected values for the analytes in these populations but rather to highlight areas where interpretation/interpretability might be compromised by unreliability and/or failures of modeling assumptions (such as heteroscedasticity and timesensitive variability). As noted earlier, this study sought to quantify the agreement between the two measurement methods and/or their disagreement, and not to quantify or correct for the degree of disagreement. The magnitudes of the differences our analyses discovered could be clinically relevant. For example, in the evaluation of adrenal insufficiency they could make the difference between concluding someone has adrenal insufficiency or adrenal sufficiency. In this particular cohort of patients, $11.5 \%$ of those tested could be given a different diagnosis (adrenal insufficiency versus adrenal sufficiency) depending on which assay was used to make the diagnosis. Similarly, the differences between the thyroid hormone assays are clinically relevant, particularly in the pregnant population where the thyroid hormone concentration could be most relevant for fetal health [11-13]. Here, our samples did not permit distinction of diagnosis on the basis of IA or MSMS, but this has been found in a current example of FT4 results where 5/46 (10.9\%) of patients had IA results for FT4 that could have led to misdiagnosis [20].

Our results suggest that immunoassay and tandem mass spectrometry cannot be considered to yield interchangeable results. The methods did not agree and this disagreement became more extreme and less predictable at higher concentrations of the analytes we studied. As the true concentration becomes more extreme, so does the discrepancy between IA and MSMS results. The implication of this for clinicians is that patients with analyte values at the extremes are more likely to be misdiagnosed/mismeasured when IA is used (see $[17,20,24,25]$.

Since the extreme observations are by definition rare outcomes, they might appear to be outliers and not contribute much (particularly in larger samples) to decrease the $R^{2}$. This can artificially inflate confidence in overall fit of a transformation to the data and can lead to undetected incorrect clinical decisions if IA is used and homoscedasticity is assumed.

Our results demonstrate good agreement between IA and MSMS in the concentration range of least interestthat is, at normal levels [20, 23-26, 29]. More importantly, the methods do not give similar results over time and over the actual concentration of cortisol and, to a lesser but still "genuine" extent, FT4. MSMS has been compared to equilibrium dialysis [25] for analytes including FT4 with excellent agreement.

IA and MSMS methods have been compared elsewhere [17-20], suggesting that IA methods lack specificity and are also subject to interferences from altered binding proteins and nonspecific heterophilic antibodies-which are prevalent during pregnancy (possibly contributing to some of our results). Direct analogue FT4 IA methods have been found to give false results at both the low and high ends of the value continuum [17-20]. Different serum matrices can affect IAs but are far less likely to affect MSMS methods. The latter do not cross-react with similar compounds or metabolites, and so are more specific. Immunoassays, in contrast, may cross-react with similar compounds as well as with many metabolites. The precision or reproducibility of MSMS methods and IA methods are similar, although some IA methods could repeatedly provide a precise, and incorrect value. Drawbacks to MSMS methods are that they require more highly trained operators and generally are slower, with a lower throughput than IAs. Several university, hospital and commercial laboratories are moving from the non-specific immunoassay platforms to the more specific tandem mass spectrometry procedures for steroid and thyroid hormone measurement. This trend is accelerating and the number of laboratories using tandem MSMS and participating in the College of American Pathologists Proficiency Testing Program is increasing significantly each year.

In conclusion, these analyses demonstrate statistically significant disagreement in the measurement of two analytes at levels outside of the reference range by two different assays. It is important for physicians who are making clinical decisions to be aware that the analyte value they are provided with varies depending on the assay used to generate the data. Clinicians may be surprised to discover that the clinical decision they reach may be impacted by the assay employed. The mechanism of action of the MSMS assay relative to that of IA methods support MSMS as the more specific and accurate assay (see also $[14,16-20]$ ). Since disagreement is most pronounced at the extremes (highest/lowest) and 
TABle 3: Pearson correlations of difference between methods versus MSMS alone, for Free T4 (FT4). MSMS and (MSMS-IA) correlations reflect significant differences in the variances of the measurements by IA versus MSMS over time, and for nonpregnant women (NP) measured at one time only. (Pregnant cohort $\mathrm{N}$ with both ranges from 59 (T1) to 26 (T3); Nonpregnant cohort $\mathrm{N}$ is 28. )

\begin{tabular}{lllll}
\hline DIFFERENCE & \multicolumn{3}{c}{ FT4 by MSMS, over time (and for NP) } \\
& FT4 by MSMS, T1 & FT4 by MSMS, T2 & FT4 by MSMS, T3 & FT4 by MSMS, NP \\
\hline FT4, T1 MSMS-IA & $0.598 \dagger^{*}$ & & - \\
FT4, T2 MSMS-IA & & $0.823 \dagger^{*}$ & $0.723 \dagger^{*}$ & - \\
FT4, T3 MSMS-IA & & - & - \\
FT4, NP MSMS-IA & - & - & $0.411 \dagger^{*}$ \\
\hline
\end{tabular}

† Pearson correlation coefficient significant at $P<.05 .{ }^{*}$ indicates significant differences in the variances of the measurements by IA versus MSMS.

clinical intervention is typically based on values in these ranges, we feel that MSMS is the more robust method for assay.

\section{Acknowledgments}

Dr. R. Tractenberg was supported in part by Grant M01RR1329 from the National Center for Research Resources and in part by Grant K01AG027172 from the National Institute on Aging. Dr. S. Soldin is partially supported by NIH GCRC Grant no. MO1-RR-020359, by Grant 1 U10HD45993-02 of the National Institute of Child Health and Development and partially by Applied Biosystems/Sciex. Dr. J. Jonklaas is supported by National Center for Research Resources Grant K23 RR16524. The authors thank Dr. Niek Verwey for helpful comments on the paper.

\section{References}

[1] J. C. Nunnally and I. H. Bernstein, Psychometric Theory, McGraw-Hil, New York, NY, USA, 3rd edition, 1994.

[2] D. G. Altman and J. M. Bland, "Measurement in medicine: the analysis of method comparison studies," The Statistician, vol. 32, pp. 307-317, 1983.

[3] J. M. Bland and D. G. Altman, "Measuring agreement in method comparison studies," Statistical Methods in Medical Research, vol. 8, no. 2, pp. 135-160, 1999.

[4] J. M. Bland and D. G. Altman, "Statistical methods for assessing agreement between two methods of clinical measurement," Lancet, vol. 1, no. 8476, pp. 307-310, 1986.

[5] P. D. Oldham, Measurement in Medicine: The Interpretation of Numerical Data, Lippincott Press, Philadelphia, Pa, USA, 1968.

[6] J. M. Bland and D. G. Altman, "A note on the use of the intraclass correlation coefficient in the evaluation of agreement between two methods of measurement," Computers in Biology and Medicine, vol. 20, no. 5, pp. 337-340, 1990.

[7] J. M. Bland and D. G. Altman, "Comparing methods of measurement: why plotting difference against standard method is misleading," Lancet, vol. 346, no. 8982, pp. 1085-1087, 1995.

[8] D. S. Cooper, Ed., Medical Management of Thyroid Disease, Marcel Dekker, New York, NY, USA, 2001.

[9] G. P. Chrousos and A. N. Margioris, Eds., Adrenal Disorders, Humana Press, Totowa, NJ, USA, 2001.

[10] S. M. Adler and L. Wartofsky, "The nonthyroidal illness syndrome," Endocrinology and Metabolism Clinics of North America, vol. 36, no. 3, pp. 657-672, 2007.
[11] G. Morreale De Escobar, M. J. Obregon, and F. Escobar Del Rey, "Is neuropsychological development related to maternal hypothyroidism or to maternal hypothyroxinemia?" Journal of Clinical Endocrinology and Metabolism, vol. 85, no. 11, pp. 3975-3987, 2000.

[12] V. J. Pop, J. L. Kuijpens, A. L. Van Baar et al., "Low maternal free thyroxine concentrations during early pregnancy are associated with impaired psychomotor development in infancy," Clinical Endocrinology, vol. 50, no. 2, pp. 149-155, 1999.

[13] V. J. Pop, E. P. Brouwers, H. L. Vader, T. Vulsma, A. L. Van Baar, and J. J. De Vijlder, "Maternal hypothyroxinaemia during early pregnancy and subsequent child development: a 3-year followup study," Clinical Endocrinology, vol. 59, no. 3, pp. 282-288, 2003.

[14] L. J. Kricka, "Interferences in immunoassay—still a threat," Clinical Chemistry, vol. 46, no. 8, pp. 1037-1038, 2000.

[15] A. H. B. Wu, "Quality specifications in thyroid diseases," Clinica Chimica Acta, vol. 346, no. 1, pp. 73-77, 2004.

[16] G. G. Klee, "Human anti-mouse antibodies," Archives of Pathology \& Laboratory Medicine, vol. 124, pp. 921-923, 2000.

[17] S. J. Soldin, N. Soukhova, N. Janicic, J. Jonklaas, and O. P. Soldin, "The measurement of free thyroxine by isotope dilution tandem mass spectrometry," Clinica Chimica Acta, vol. 358, no. 1-2, pp. 113-118, 2005.

[18] B. W. Steele, E. Wang, G. G. Klee et al., "Analytic bias of thyroid function tests: analysis of a College of American Pathologists fresh frozen serum pool by 3900 clinical laboratories," Archives of Pathology and Laboratory Medicine, vol. 129, no. 3, pp. 310$317,2005$.

[19] M. d'Herbomez, G. Forzy, F. Gasser, C. Massart, A. Beaudonnet, and R. Sapin, "Clinical evaluation of nine free thyroxine assays: persistent problems in particular populations," Clinical Chemistry and Laboratory Medicine, vol. 41, no. 7, pp. 942947, 2003.

[20] J. Gu, O. P. Soldin, and S. J. Soldin, "Simultaneous quantification of free triiodothyronine and free thyroxine by isotope dilution tandem mass spectrometry," Clinical Biochemistry, vol. 40, no. 18, pp. 1386-1391, 2007.

[21] T. Guo, M. Chan, and S. J. Soldin, "Steroid profiles using liquid chromatography-tandem mass spectrometry with atmospheric pressure photoionization source," Archives of Pathology and Laboratory Medicine, vol. 128, no. 4, pp. 469-475, 2004.

[22] T. Guo, R. L. Taylor, R. J. Singh, and S. J. Soldin, "Simultaneous determination of 12 steroids by isotope dilution liquid chromatography-photospray ionization tandem mass spectrometry," Clinica Chimica Acta, vol. 372, no. 1-2, pp. 7682, 2006. 
[23] S. J. Soldin, C. Brugnara, and C. Wong, Eds., Pediatric Reference Ranges, AACC Press, Washington, DC, USA, 6th edition, 2007.

[24] J. P. Holst, S. J. Soldin, R. E. Tractenberg et al., "Use of steroid profiles in determining the cause of adrenal insufficiency," Steroids, vol. 72, no. 1, pp. 71-84, 2007.

[25] N. Kahric-Janicic, S. J. Soldin, O. P. Soldin, T. West, J. Gu, and J. Jonklaas, "Tandem mass spectrometry improves the accuracy of free thyroxine measurements during pregnancy," Thyroid, vol. 17, no. 4, pp. 303-311, 2007.

[26] J. Gu and S. J. Soldin, "Simultaneous quantification of free triidothyronine and free thyroxine by tandem mass spectrometry," Clinical Biochemistry, vol. 53, no. 6, p. D99, 2007.

[27] M. E. May and R. M. Carey, "Rapid adrenocorticotropic hormone test in practice. Retrospective review," American Journal of Medicine, vol. 79, no. 6, pp. 679-684, 1985.

[28] P. F. Speckart, J. T. Nicoloff, and J. E. Bethune, "Screening for adrenocortical insufficiency with cosyntropin (synthetic ACTH)," Archives of Internal Medicine, vol. 128, no. 5, pp. 761763, 1971.

[29] O. P. Soldin, M. Jang, T. Guo, and S. J. Soldin, "Pediatric reference intervals for free thyroxine and free triiodothyronine," Thyroid, vol. 19, no. 7, pp. 699-702, 2009. 


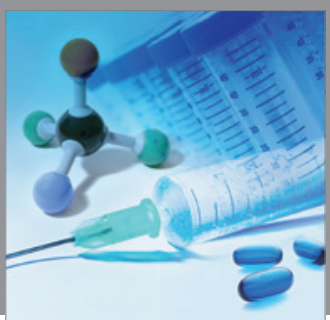

International Journal of

Medicinal Chemistry

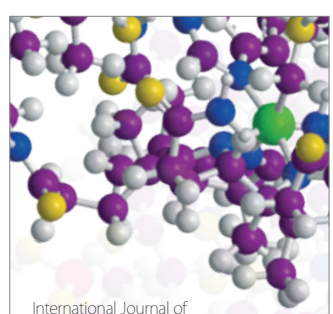

Carbohydrate Chemistry

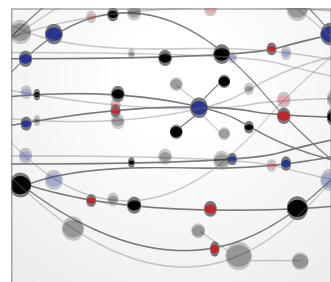

The Scientific World Journal
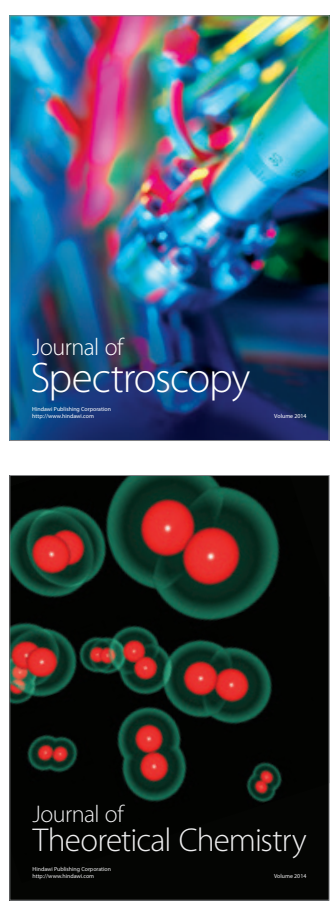
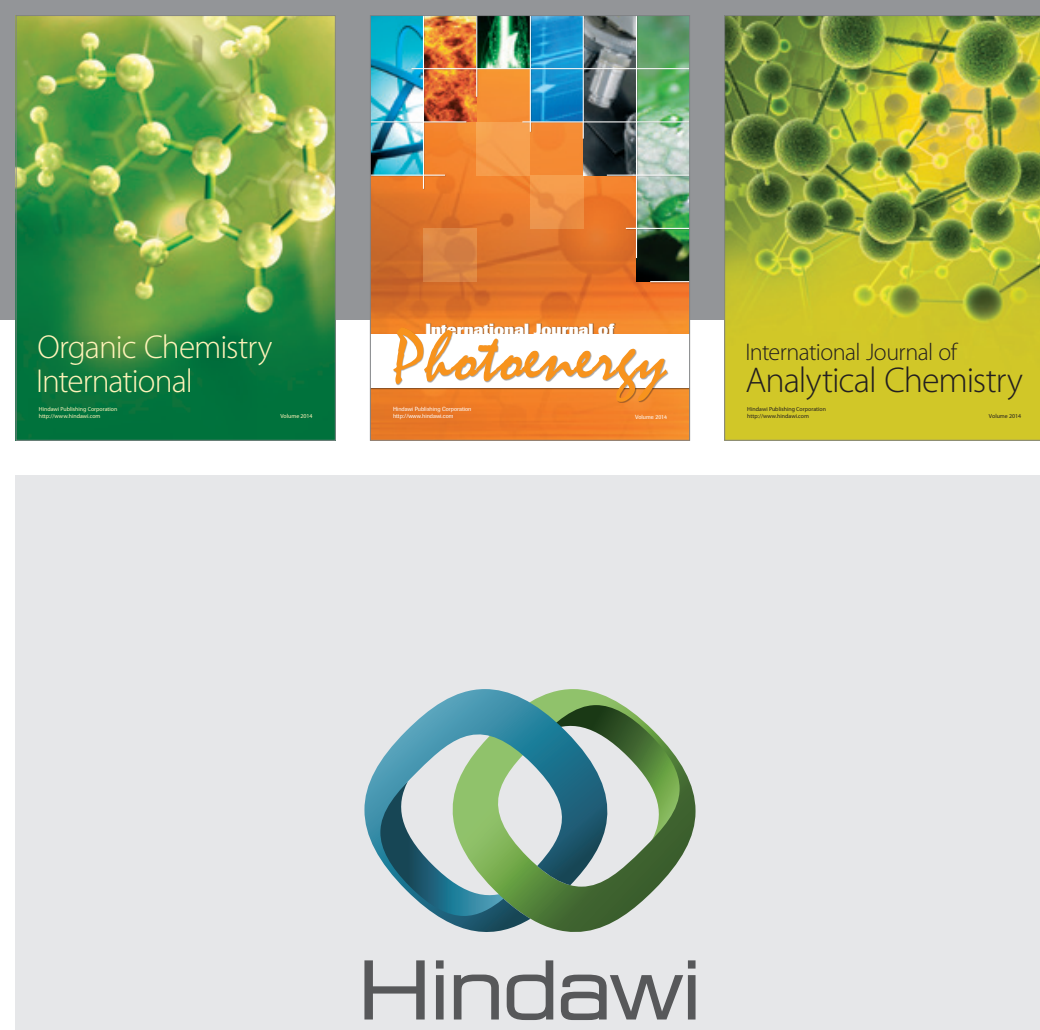

Submit your manuscripts at

http://www.hindawi.com
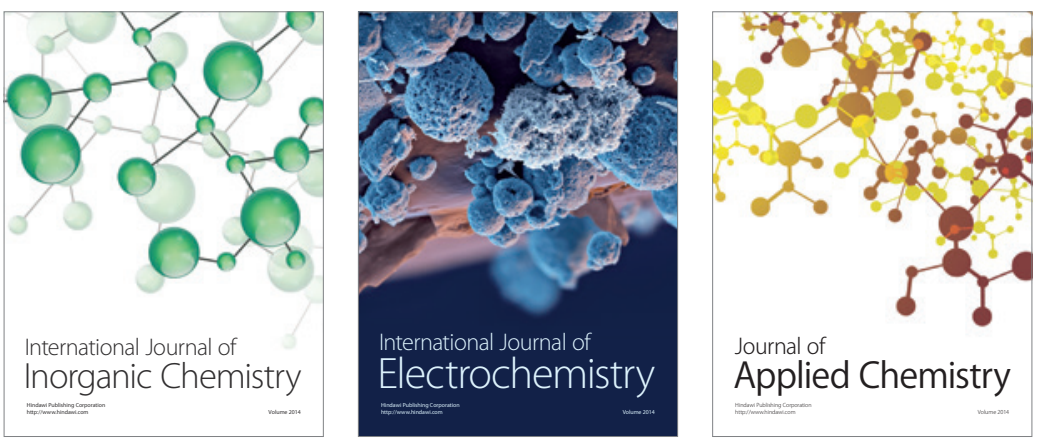

Journal of

Applied Chemistry
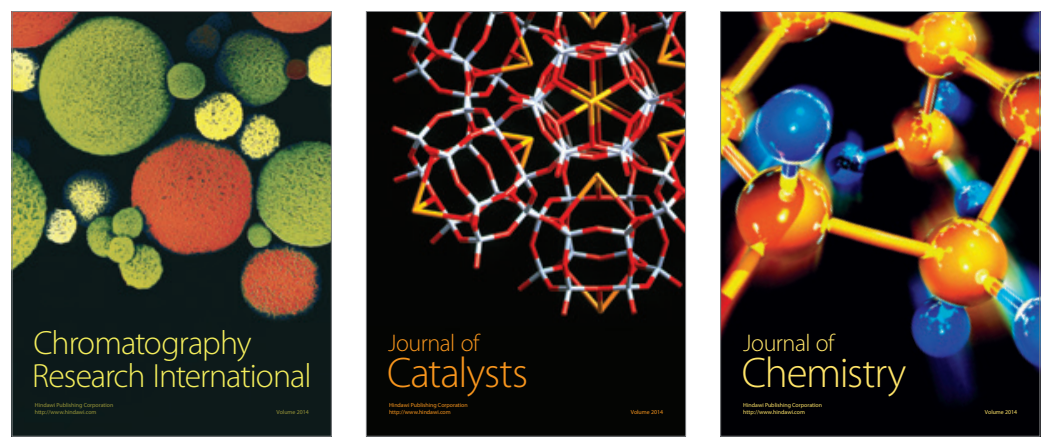
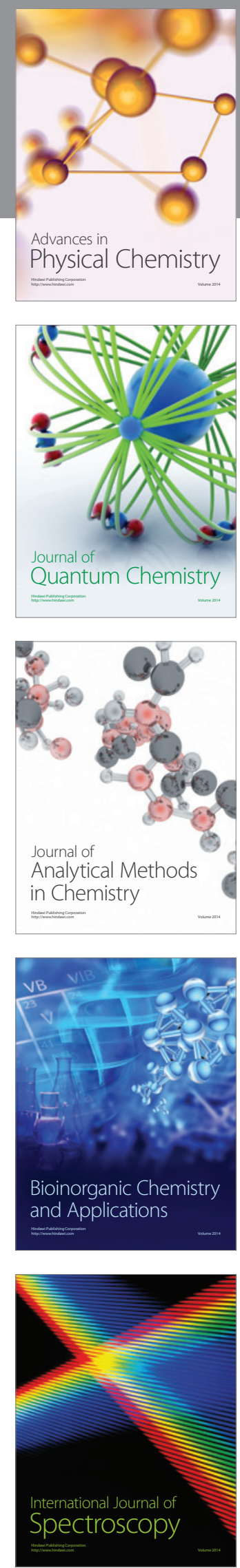